\title{
Modelling afterslip processes on a strike-slip fault in the framework of a non-singular crack theory
}

\author{
Maria Elina Belardinelli and Maurizio Bonafede \\ Dipartimento di Fisica, Settore di Geofisica, Università di Bologna, Italia
}

\begin{abstract}
A two-dimensional, quasi-static, open to the Earth surface crack is considered to model postseismic shallow creep. The gouge of a transcurrent vertical fault is modeled as a viscoplastic layer with Bingham rheology. Creep may occur on the superficial fault portion for stress values greater than a plastic threshold of the fault gouge. Seismic slip is assumed to generate a two-level stress distribution: in the deeper level, it causes the total stress to drop to values less than the plastic threshold, while, in the shallower level, it concentrates stress to values greater than the plastic threshold. Initially the creeping region extends from the Earth surface to the depth of transition between stress levels but subsequently its deeper penetration occurs thanks to stress concentration created by the crack beyond its tip. Slip at the Earth surface is computed for some stress drop distributions and viscosity profiles and the results are compared with afterslip observations on three California faults. After removal of the first afterslip phase, probably linked to the superficial local properties of fault regions, the three afterslip events look very similar to each other. In particular, a suitable choice of the stress parameter values gives a good data reproduction in all the analysed cases even if the viscosity is assumed to be uniform on the fault plane. These parameter values are consistent with a temporal deepening of the creeping region even if the computed diplacement at the Earth surface along a direction orthogonal to the fault strike is scarcely sensitive to maximum depth of creep.
\end{abstract}

\section{Introduction}

The aseismic fault slip or creep process which occurs after an earthquake and implies a final slip of the order of the maximum coseismic slip, is called «afterslip». The process is episodic, that is, not stationary and in particular it is characterized by a slip rate decay to null values in a time interval of the order of months or years. Creep processes were observed on the upper part of active faults, since they are directly visible at the Earth surface either by means of alignment arrays, whose distances are periodically remeasured (e.g., Louie et al., 1985; Sharp and Saxton, 1989), or by means of continuous measurements through creepmeters (e.g., Smith and Wyss, 1968; Cohn et al., 1982; Bilham , 1989), or by means of non-instrumental measurements including the direct measurement of the dislocation on man-made features that cross the fault, such as roads, fences, curbs (e.g., Allen and Smith, 1966). The continuous recording through creepmeters allowed one to identify a detail structure in the superficial creep processes. This structure consists of a temporal sequence of elementay creep events: brief episodes during which the slip velocity increasing and its subsequent extinction occur. The typical temporal scale of an elementary creep event ranges from minutes to hours and the developed slip is of the order of magnitude of millimeters. Such events may occur individually and without correlation with earthquakes. Creep events and afterslip are often sumperimposed to «secular creep» which has a typical linear temporal evolution and a clear tectonic origin. 
The episodic character of the creep processes can be explained if they are regarded as threshold phenomena, that is, if it is assumed that the fault gouge material undertakes an anelastic deformation only if the applied stress is greater than a certain value, which is often interpreted as plastic threshold (Nason and Weertman, 1973). Rather complicate constitutive relationships were used to describe the threshold dependence or the sliding resistance dependence on time and on the state variables. Nevertheless, the point of many proposed models was mainly to be consistent with the observations starting from the simplest fault gouge constitutive relationships. In order to reproduce the observed evolution of single creep events, temporal laws as the exponential (Nason and Weertmann, 1973) or the power law (e.g., Crough and Burdford, 1977) were adopted. In Belardinelli and Bonafede (1991) the inadequacy of the exponential decreasing law for the reproduction of some observed afterslip processes is shown; besides, in his model, where a quasi-plastic rheology for the fault gouge and a power law as constitutive relation are adopted, Wesson (1988) infers that the most adequate exponent for afterslip processes is greater than the most adequate one to reproduce single creep events. From this difference between the parameters which represent each of the two processes Wesson infers that they probably have different nature: the single creep events do not involve more than the first kilometers of depth, while the afterslip, that is a longer-term process, would concern a zone extending to a greater depth. To reproduce in particular afterslip processes empirical evolution laws like rational versus time law (Sharp and Saxton, 1989; William and Magistrale, 1989) or linear versus time logarithm law (Smith and Wyss, 1968, Chon et al., 1982) were suggested. According to William and Magistrale (1989) the advanced afterslip phases after the Superstition Hills earthquake may be reproduced using a logarithmic law, while the faster initial phases, during the early several weeks, are better described by a power law. Nevertheless Bilham (1989) notes that both these two time functions are not compatible with the asymptotic stationary slip value attainment which can be recovered after eventually detrending the secular slip component from data. From the models by Belardi- nelli and Bonafede (1991) and by Wesson (1988) one deduces that the initial phases of the slipping process are strongly dependent on the adopted fault gouge rheological parameters, while the maximum final slip does not depend on them. If Wesson obtains a poor fit for the initial phases of the process, Belardinelli and Bonafede, assuming a vertically heterogeneous viscoplastic rheology, obtain a satisfactory reproduction of the whole process in all the examined cases.

In cases in which the afterslip process can be directly observed at the Earth surface, one can estimate the depth extension of the creeping zone by geodetic measurements both at great and small distance from the fault (Wesson, 1988). In particular, from these observations it can be inferred that the afterslip often does not involve much more than the first kilometers in depth on the fault and can eventually yield a slip at the surface equal to the coseismic slip deficiency which occurs in this zone during a deep earthquake (Scholz et al., 1969). For this reason several authors modelled afterslip processes assuming that these phenomena concerned a superficial fault region with a rigid boundary at depth, that is, they assumed that the aseismic region were adjacent but separated from the deeper one where the seismic dislocation took place (e.g., Scholz et al., 1969; Wesson, 1988; Belardinelli and Bonafede, 1991; Scholz, 1991). In the previous models, the confined creeping zone constraint introduces a singularity in the stress field created by the aseismic crack.

In the present work a quasi-static and non-singular crack model is adopted following the theory developed by Bonafede et al. (1985) and Bonafede and Dragoni (1987). At the beginning the aseismic crack extends down to the depth beyond which the seismic region extends. In the zone dislocated by the earthquake, a creep resistance asperity equal to the difference between the residual earthquake stress field plus the crack selfstress field and the yield threshold is considered. During the earthquake in fact a stress drop takes place in the deeper fault region, so that the earthquake stress field opposes the aseismic crack extension at depth. Nevertheless crack can extend thanks to the self-stress concentration at its tip. The creeping region propagates inside different height asperity down to depths which are 
much greater than the initial extension of the creeping zone, even if the region where a significant slip takes place remains confined to shallow depth.

After the analysis of the effect of small viscosity variations passing from the initially not dislocated zone to the one dislocated by the earthquake, we make the assumption that after a very fast initial slippage, the process develops as if the viscosity was uniform with depth and was mainly conditioned by the initial stress distribution on the fault plane. The very fast initial slip, that is scarcely constrained by data, is introduced in the model as a free parameter. The model is then tested by the comparison with afterslip data from which a suitable initial displacement was subtracted (Allen and Smith, 1966; Sharp and Saxton, 1989, Louie, 1991, written communication).

\section{The model}

Suppose that on a strike-slip fault, unbounded along the fault strike, a seismic slip with consequent stress redistribution occurs at depth.

We consider that the post-seismic stress is a two-level function of depth: one in the superficial zone, above the region which slipped seismically, where the seismic slip concentrates stress; the other lower than the previous one, in the deeper region where an external stress drop oc- curs with the earthquake. The aseismic fault zone is described by a quasi-static, two-dimensional crack with fracture surface $y=0$, in the elastic half-space $x>0$ with rigidity $\mu$ in antiplane stress configuration. The crack is open to the free surface $x=0$ and extends down to the depth $x=a$ (fig. 1a)). We then assume a screw dislocation distribution with a time-dependent density $D(x, t)$, such that the displacement discontinuity $\Delta u(x, t)$ is

$$
\Delta u(x, t)=B \int_{-a}^{x} D\left(x^{\prime}, t\right) \mathrm{d} x^{\prime}
$$

with $B$ dislocation distribution Burgers vector module. The crack tip evolution $a=a(t)$ is obtained as part of the solution together with the displacement discontinuity at the free surface $\Delta u(x=0, t)$. The crack tip dynamics in particular is obtained from the non-singular stress condition at the same crack tip, according to Bonafede and Dragoni (1987) method. The equation for the density $D(x, t)$ is, in each instant, the equilibrium equation among the stresses acting on the slipping surface:

$$
\begin{gathered}
q(x, t)=s_{\infty}+s_{e}(x)-\sigma_{v}(x, t)-Y(x), \\
|x|<a, t>0
\end{gathered}
$$

where $q(x, t)$ is the transient stress drop on the crack surface, $s_{\infty}$ is the ambient stress, $s_{e}$ is the

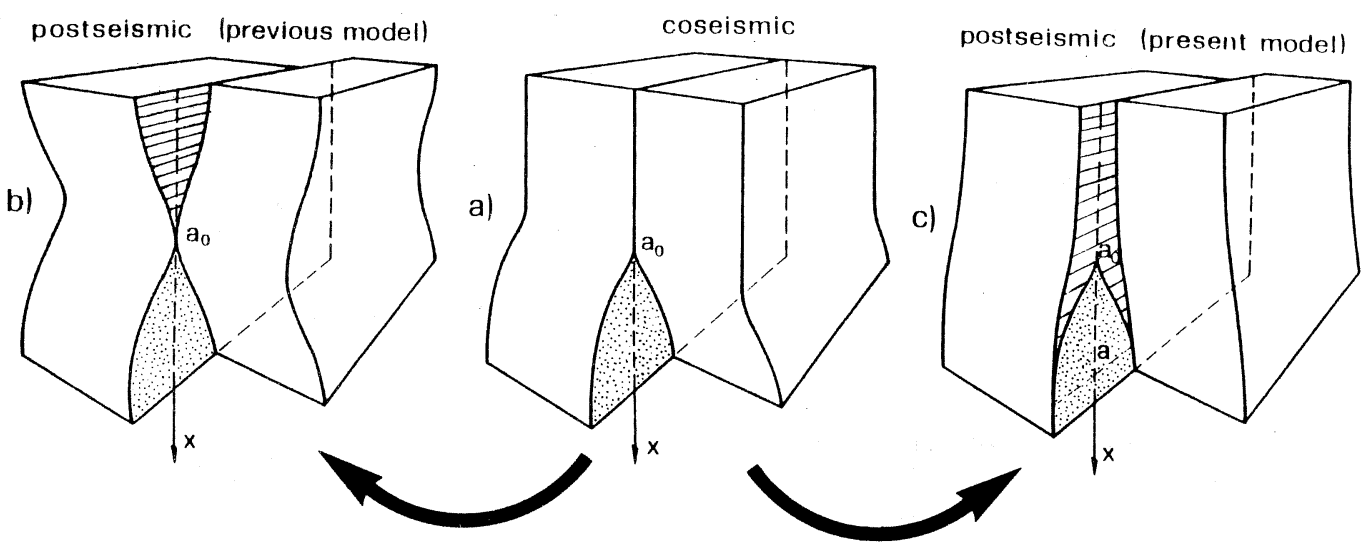

Fig. 1. Representation of the regions with seismic (dotted) and aseismic (dashed) slip on a strike-slip ( $x$, depth): a) at the earthquake instant, b) after the earthquake in our 1991 model, c) after the earthquake in the present model. 
stress created by the seismic slip at depth, $Y(x)$ is a plastic threshold and $\sigma_{v}(x, t)$ is a viscous stress which opposes the slip. To obtain $\sigma_{v}(x, t)$ we assume that the fault gouge has a viscoplastic behaviour, so that the slip occurs with linear relation between stress and strain velocity for greater stress values than the plastic threshold $Y(x)$. In this way we have

$\sigma_{v}(x, t)=\frac{\eta(x)}{2 w} \Delta \dot{u}(x, t)$, if $s_{\infty}+s_{e}(x)>Y(x)$

where $\eta(x)$ is the viscosity of the zone near the fault plane, which undergoes plastic deformation. This zone is modeled as a vertical layer with thickness $w$, embedded between two elastic halfspaces with rigidity $\mu$.

We satisfy the free surface condition at $x=0$ by extending symmetrically, with respect to $x=0$, the stress drop $\sigma(x)$ and the viscosity $\eta(x)$ and searching for the solution $D(x, t)=-D(-x, t)$ of the extended problem. The restriction of such a solution to the half-space $x>0$ is automatically the solution of the original problem which satisfies the required boundary conditions. In the case of the extended problem one has (e.g., Bilby and Eshelby, 1968)

$$
q(x, t)=\frac{\mu B}{2 \pi} \int_{-a}^{a} \frac{D\left(x^{\prime}, t\right)}{x-x^{\prime}} \mathrm{d} x^{\prime}
$$

In the following we assume

$$
\begin{aligned}
& \sigma(x)=\frac{s_{\infty}+s_{e}(x)-Y(x)}{\mu}=\left\{\begin{array}{c}
S,|x|<a_{0} \\
-r_{s} S,|x|>a_{0}
\end{array}\right. \\
& \gamma(x)=\frac{\eta(x)}{\eta_{0}}=\left\{\begin{array}{l}
r_{v},|x|<a_{0} \\
1,|x|>a_{0}
\end{array}\right.
\end{aligned}
$$

where $r_{s}$ and $r_{v}$ are two positive dimensionless parameters, $S$ is a positive constant stress, and $\eta_{0}$ is the maximum value of $\eta(x)$. As far as the effects on the slip at the surface as a function of time are concerned we can assume that the crack yields a uniform stress field on the aseismic region. In fact in Belardinelli and Bonafede (1991) it is shown that $\Delta u(0, t)$ does not vary sensitively if a heterogeneous stress profile on the aseismic region instead of a uniform one is used.

While in the 1991 paper we adopted an aseismic crack with fixed axtension, in the present one the aseismic crack at $t=0$ extends down to the depth $x=a_{0}$, and progressively extends into the negative stress drop region (fig. 1). The solution of eq. (2), where the quantities which are defined in (1), (3), (4) were substituted, can be found if one writes $D(x, t)$ as in the following (e.g., Bonafede and Dragoni, 1987):

$$
D(x, t)=\frac{1}{B}\left(1-\xi_{a}^{2}\right)^{-1 / 2} \sum_{n=1}^{\infty} \alpha_{n}\left(a, \tau_{a}\right) T_{n}\left(\xi_{a}\right)
$$

where $T_{n}$ is the $n$-th order Chebychev polynomial of the first kind, $\xi_{a}=x / a, \tau_{a}=t / T_{a} ; T_{a}=$ $2 \eta_{0} a /(\pi \mu w)$ is a characteristic time which depends on the maximum viscosity value $\eta_{0}$ and the index $a$ refers to the value $a(t)$ of the crack amplitude. This position tranforms the integrodifferential equation for $D(x, t)$ into an infinite system of ordinary differential equations for the coefficients $\alpha_{j}(a, \tau)$, that is, in an infinite system of algebraic equations for the Laplace transform of the coefficients; we solve this system through truncation at a finite order $M$.

It is not possible here to specify the detailed procedure we have followed, however it is drawn from the one exposed in Bonafede and Dragoni (1987). In the case of functions $\sigma(x)$ and $\gamma(x)$ which are symmetric in $x$, one can demonstrate that the non-singular stress condition at the crack tip is

$$
\sum_{n=1}^{M} \alpha_{n}\left(a, \tau_{a}\right)=0
$$

From this implicit equation the crack amplitude $a(t)$ et each instant can be determined.

The final crack amplitude $a_{f}$, which satisfies the non-singular stress condition (7) written for $\tau_{a}=\infty$, can be put theoretically for $M=\infty$ in the following exact form:

$$
a_{f}=\left[\cos \left(\frac{\pi}{2\left(1+r_{s}\right)}\right)\right]^{-1} a_{0}
$$


the same solution can be obtained also for a finite truncation order $M$, such that $M /\left(2\left(1+r_{s}\right)\right)$ is an integer. The evolution was computed using a time scale factor given by $T=2 \eta_{0} a_{0} /(\pi \mu w)$.

\section{Results and comparison with data}

The system evolution (eq. (6)) for each value of $r_{s}$ was computed using a particular truncation order $M$ so that relation (8) is exacly valid. In fig. 2 the total evolution of the aseimic slippage is shown, a uniform viscosity is assumed and two cases are considered: $r_{s}=1$ (fig. $\left.2 \mathrm{a}\right)$ ) and $r_{s}=0.05$ (fig. 2b)). In the first one we can note that the crack evolves very fast and penetrates a small depth below $a_{0}$ since the stress asperity to be exceeded is great; in the second case the asperity is reduced by two orders of magnitude and then a considerable penetration is achieved.

In fig. 3a) the slip at the Earth surface as a function of time for some values of $r_{s}$ is shown: it can be noted that in particular by decreasing $r_{s}$ mainly the last phase of the process is slowed

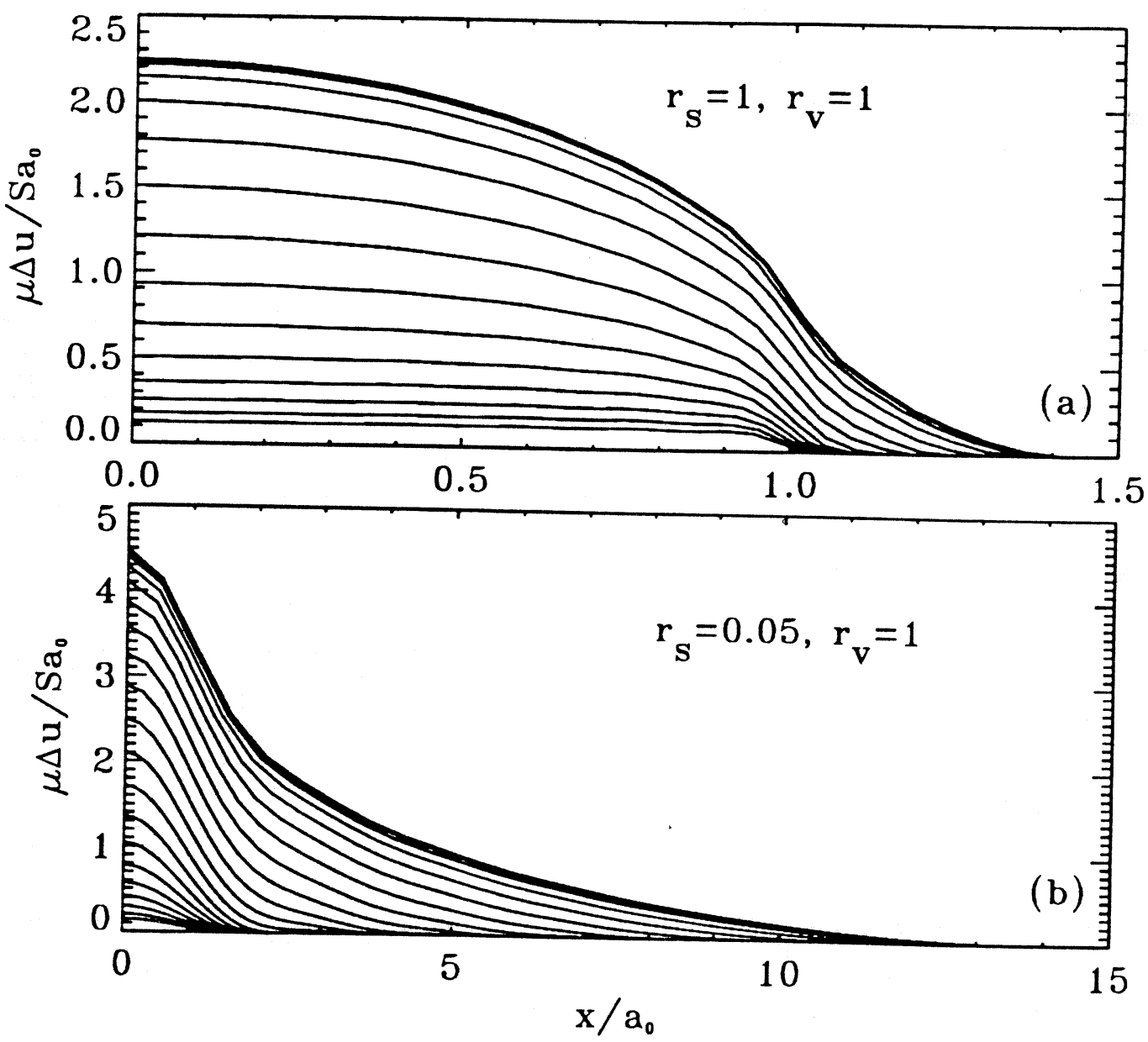

Fig. 2. Crack evolution, drawn et equal intervals in the adimensional time logarithm between $\tau=0.1$ (lowermost curve in each figure) and $\tau=1000$ (uppermost curve in each figure) for $r_{v}=1$ and for a) $r_{s}=1$ and b) $r_{s}=0.05$. Time $\tau$ is increasing from the lower curve toward the upper curve. 

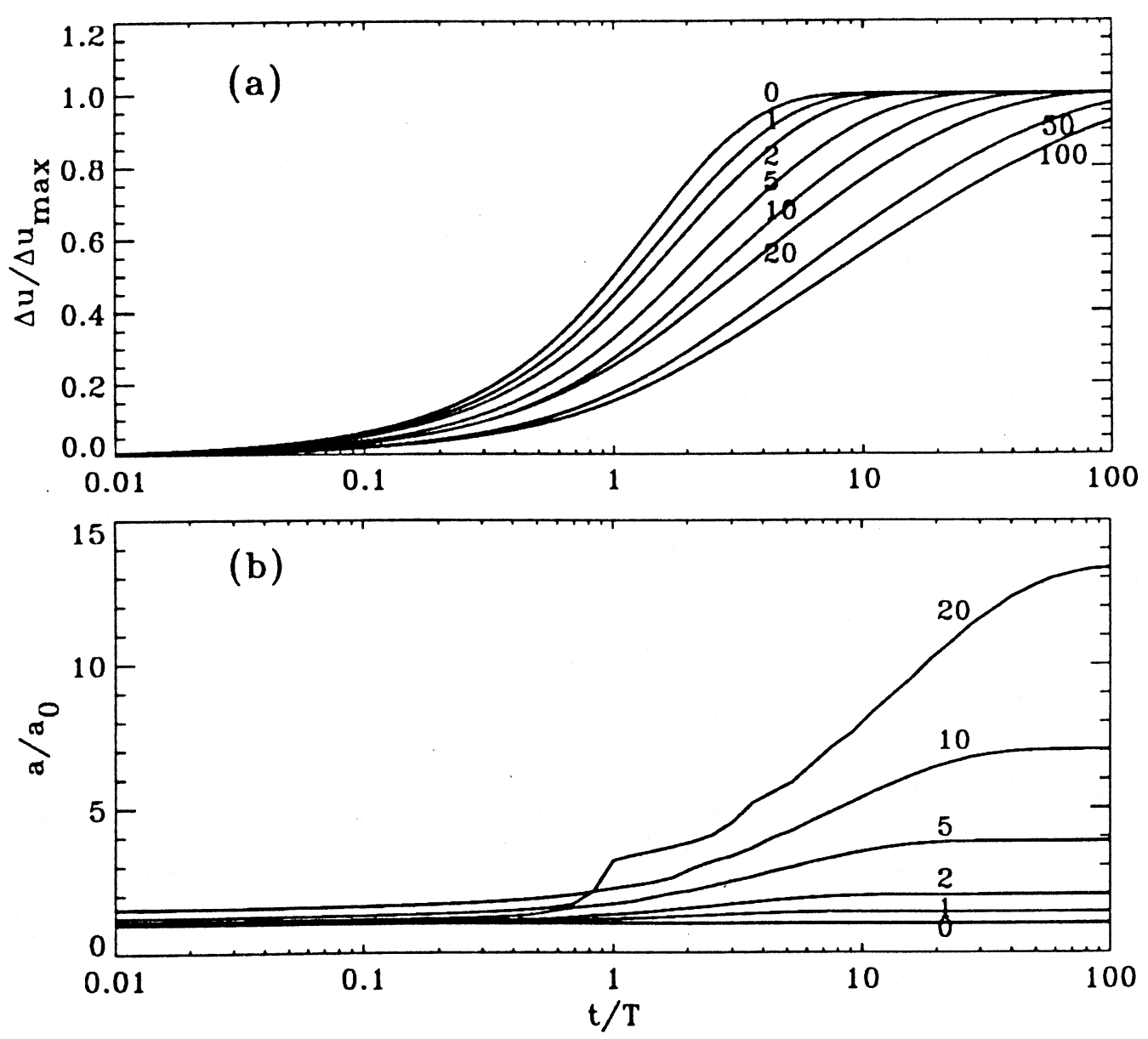

Fig. 3. a) Normalized slip at the Earth surface as a function of time and b) amplitude as a function of time with varying $r_{s}$ and $r_{v}=1$. The values next to each curve refer to $r_{s}^{-1}$.

down: in fact in this way the resistance to slip in the lower zone is decreased and the crack can penetrate a longer distance into this zone, as eq. (8) suggests, and then it employs a longer time to stop. If we instead fix $r_{s}$, by variations of $r_{v}$ in the range $0.1 \div 1$ we substantially obtain a mere time scale factor variation, that is, a translation of the curve along the logarithmic axis in the abscissa as we can see in fig. 4 . Besides in fig. $3 b$ ) it is shown that the final crack amplitude $a$ increases as $r_{s}$ decreases; some instabilities in amplitude evolution are evident, basically due to the discontinuities in the stress profiles adopted. In fact the employed input function $\sigma(x)$ imply that the coefficients $\alpha_{j}(a, \tau)$ are decreasing like $1 / j$ and then the truncated solution converges slowly. This does not affect the computed slip evolution at the Earth surface as shown in fig. 3, since this depends on the integral of $D(x, t)$ over the whole fracture surface. The $a(t)$ instability influences this integral only through a contribution which represents the slip near the crack tip, where this is very little, and then the contribution itself is negligible. Besides, owing to the oscillations due to the input functions discontinuities, we have different roots of eq. (7) for the same $r_{s}$ value: this 


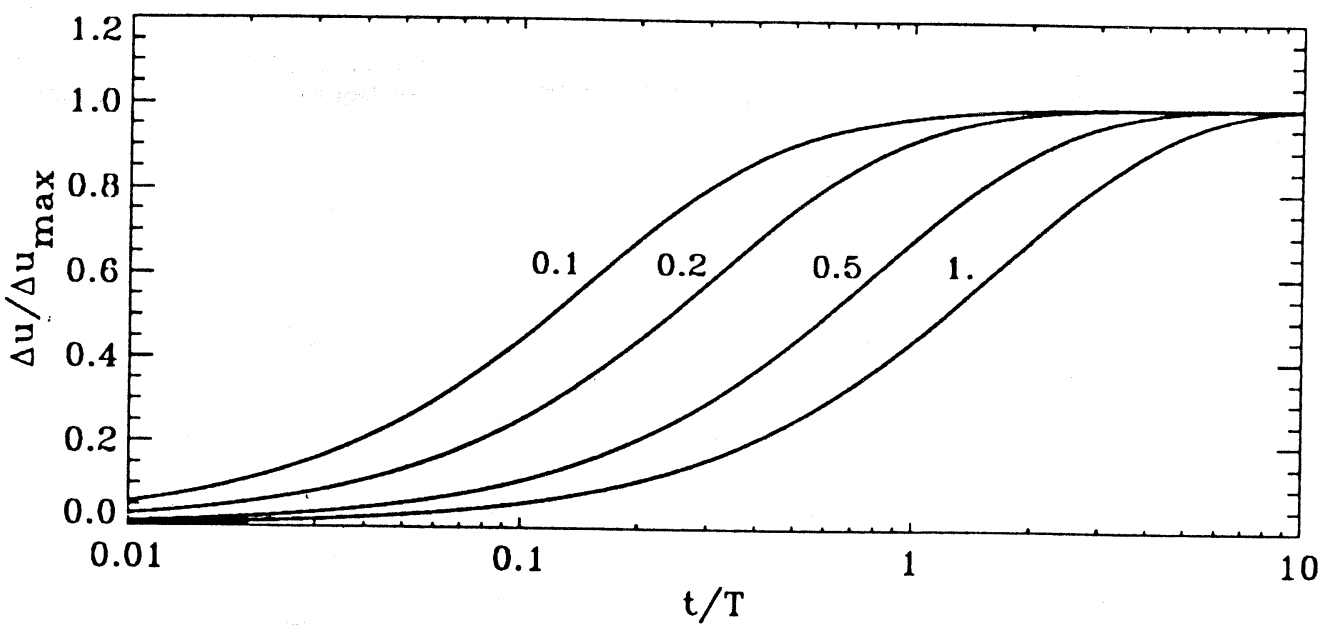

Fig. 4. Normalized slip at the Earth surface as a function of time with varying $r_{v}$ and $r_{s}=1$.

fact led us to restrict the variability range of the parameters.

Assuming that in the early phases of the process after the earthquake a very fast aseismic slip $u_{0}$ occurs and a mean viscosity $\eta_{0}$ is distributed uniformly over the whole fracture surface, the model was applied to the description of the later phases of the afterslip process. The parameters $u_{0}, T$ and $r_{s}$ were varied in order to reproduce the data (fig. 5). We deal with afterslip data which regard the Parkfield June 27th, 1966 afterslip observed through repeated measurements on the centerline of the Highway 46 (Allen and Smith, 1966), the Imperial Valley 1979 afterslip on the homonymous fault in South California, observed through repeated measurements of an alignment array near Highway 80 (Louie et al., 1985; Louie, 1991, written communication) and the Superstition Hills afterslip on the homonymous fault few kilometers North-West from the Imperial fault, measured geodetically near the locality denoted as FSSC in the paper by Sharp and Saxton (1989). In fig. 5 some type curves with varying $r_{s}$ values are shown, like those in fig. 3a), together with the three data sets. From each data set an initial suitable slip $u_{0}$ was subtracted and the set was translated along the logarithmic axis by an amount $\log T$, in order to minimize the sample standard deviation from the curve for $r_{s}=\infty$ (fig. 5a)) and from the curve for $r_{s}=0.04$ (fig. 5b)).
In fig. 5a) the first datum of each sample has been extracted, because it refers to measurements made 10-30 $\mathrm{h}$ after the earthquake and, in the case of Imperial Valley, also the last datum with respect to the sample used by Wesson (1988), since probably it is influenced by other causes different from the earthquake. In the Parkfield sample case, it is necessary to reintroduce the first datum in order to make the model self-consistent and, with decreasing $r_{s}$ to $r_{s}=0.01$, it is necessary to reintroduce also the first datum of the Superstition Hills and Imperial Valley samples. In fact, with decreasing $r_{s}$ values, the immediate postseismic slip $u_{0}$ also tends to decrease. For the Superstition Hills data we find that $r_{s}=0.04$ is the curve. The $u_{0}$ value in this case is not greater than the ratio between the slip of the second datum and final slip, and this means that the model does not predict in this case a coseismic slip at the surface, as one may estimate at least in the mid-southern part of the fault, where data were picked up (William and Magistrale, 1989). If the $r_{s}$ value is further reduced to 0.01 , The Superstition Hills data distance from the sample curve increases, but it remains quite small (standard deviation $\sim 10^{-2}$ ); on the contrary for the Imperial Valley and Parkfield data we have a little fit improvement. Nevertheless, one has to take into account that for these $r_{s}$ values the instabilities in the amplitude determination 

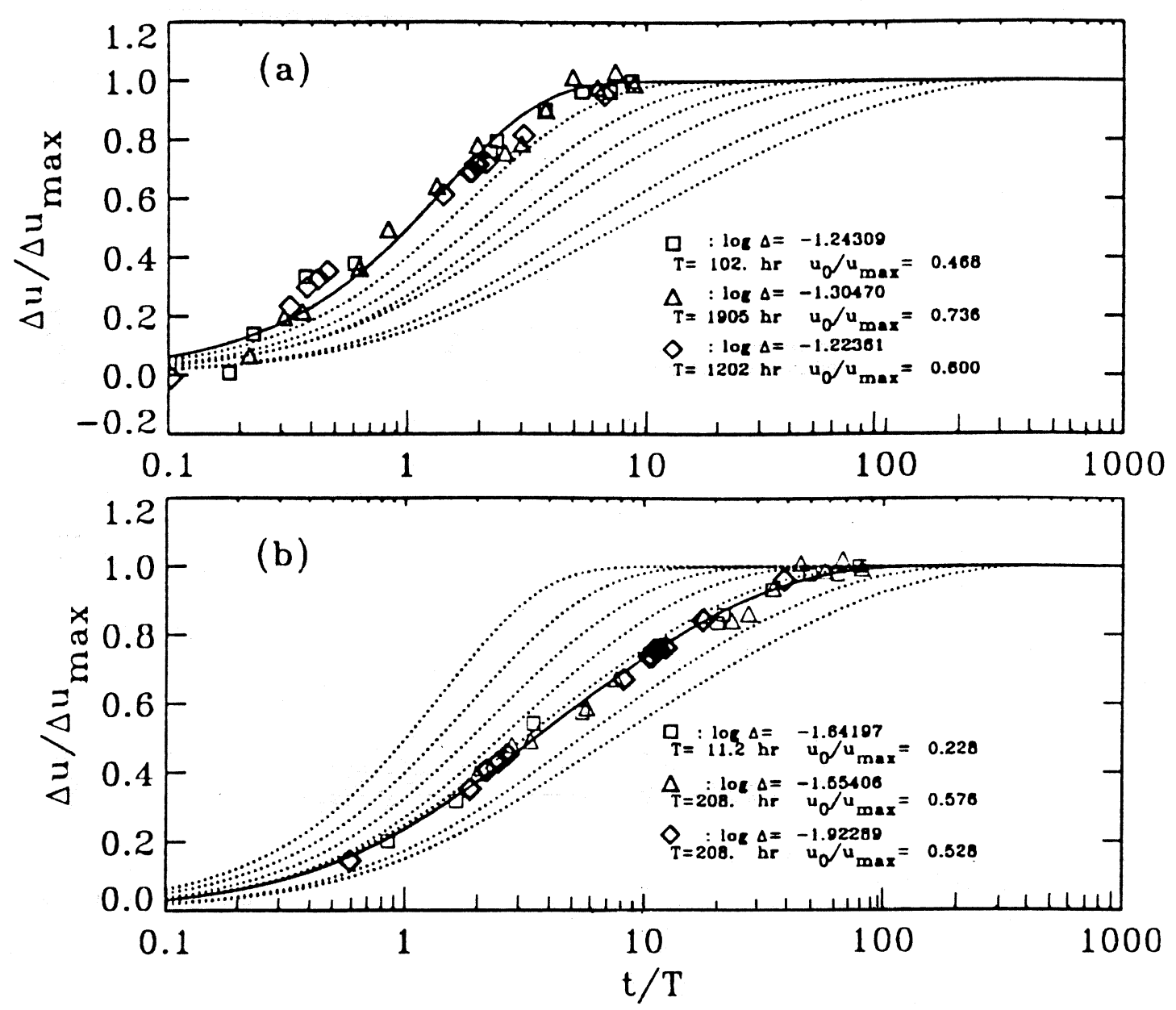

Fig. 5. Analysed data and slip evolution with varying $r_{s}$ values. Slip is normalized to the maximum value and from each sample an initial slip $u_{0}$ was subtracted, then each sample was normalized to the difference between $u_{0}$ and $u_{\max }$ and translated along the logarithmic axis by an amount equal to $\log T$ in order to realize the minimum deviation $\Delta$ from the curve with a) $r_{s}=\infty$ and b) $r_{s}=0.04$. $\diamond$ Superstition Hills, $\square$ Parkfield, $\triangle$ Imperial Valley (see text).

become significant. In any case, the Parkfield and the Imperial Valley samples show some inflexions with respect to the theoretical curve, which make the fit worse than that of Superstition Hills for any $r_{s}$ value.

Values $r_{s} \ll 1$ characterize all the three considered cases: therefore the present model suggests that, in the post-seismic phase, a progressive and noticeable depening of the creeping region occurs. In fig. 6 the evolution of displace- ment parallel to the strike along a normal direction with respect to the fault strike is shown for two different values of $r_{s}$, that is, for two final crack extensions which differ by an order of magnitude. The less is the $r_{s}$ value the wider is the distribution of displacement going away from the fault, as we can see in fig. 6 . In fig. 7 we show the asymptotic $(\tau=\infty)$ displacement at the Earth surface in a normal direction $y$ with respect to the fault strike for a non-singular crack in the best- 

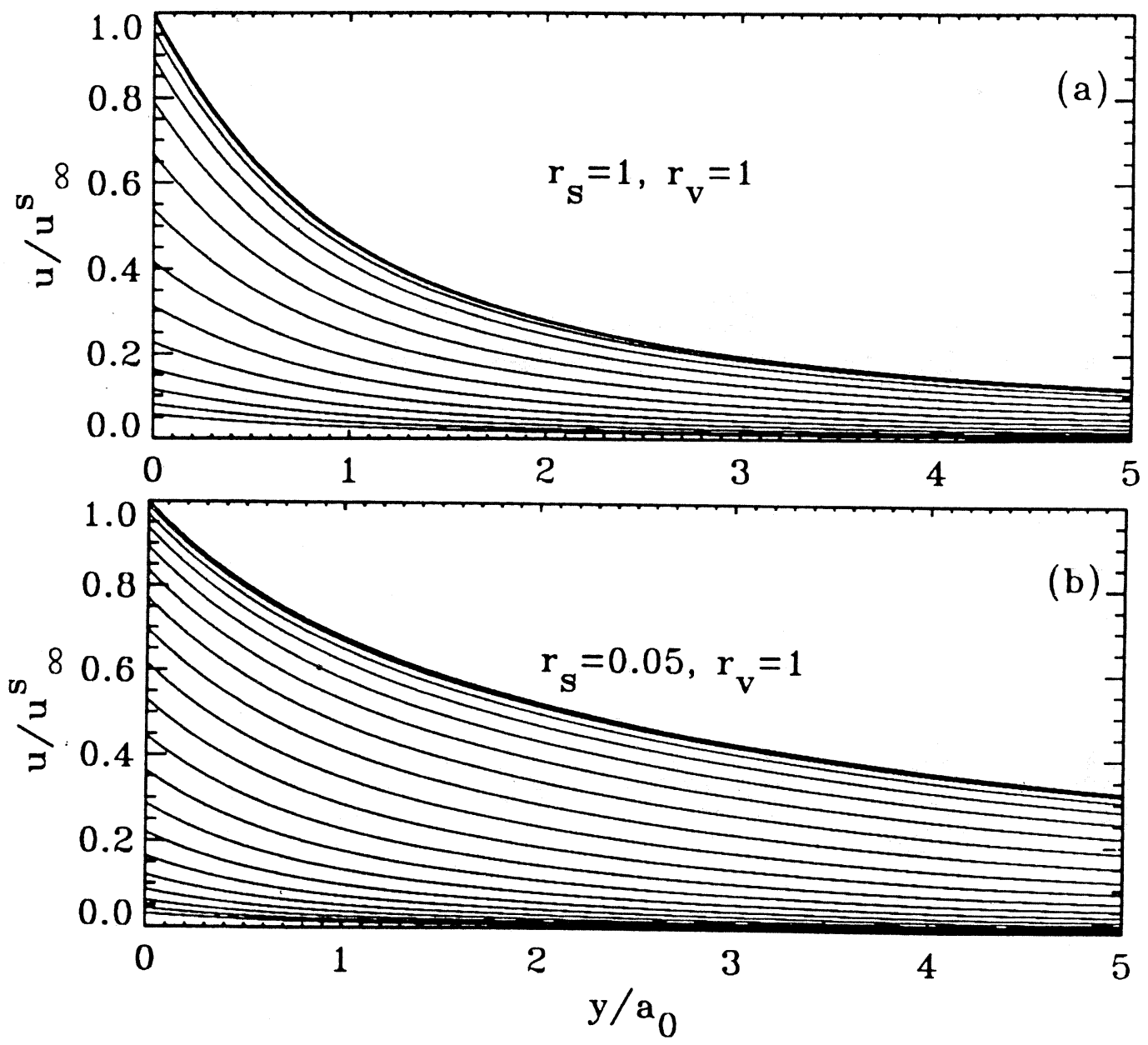

Fig. 6 Evolution of the ground displacement along the transversal direction with respect to the fault strike for a) $r_{s}=1$ and b) for $r_{s}=0.05$. $u_{\infty}^{s}$ is the displacement on the strike for $\tau=\infty$. Time $\tau$ is increasing as in fig. 2 .

fitting case of Superstition Hills and for some singular cracks $\left(r_{s}=\infty\right)$ of different amplitudes. Of course the normalized displacement in the singular case is self-similar with the distance from the fault; in fact we obtain it from

$$
u(y)=\frac{S a}{\mu}\left[\left(1+\frac{y^{2}}{a^{2}}\right)^{1 / 2}-\frac{y}{a}\right]
$$

In fig. 7 then we make a comparison between the asymptotic displacement in the present model and in our previous model where the creeping region is confined in the superficial part of the fault and a singular crack is adopted to model it. The displacement in the non-singular case near the fault is very similar to the displacements in case of singular, shallow cracks with amplitude equal to $2.2 a_{0}$ and going away from the fault the displacement tends to that created by a singular crack with amplitudes $4.6 a_{0}$. From this comparison we may say that we could not appreciate the effective depth to which the creeping region is extended using field observation of displacement at various distances from the fault since the developed displacement is very similar to that pro- 


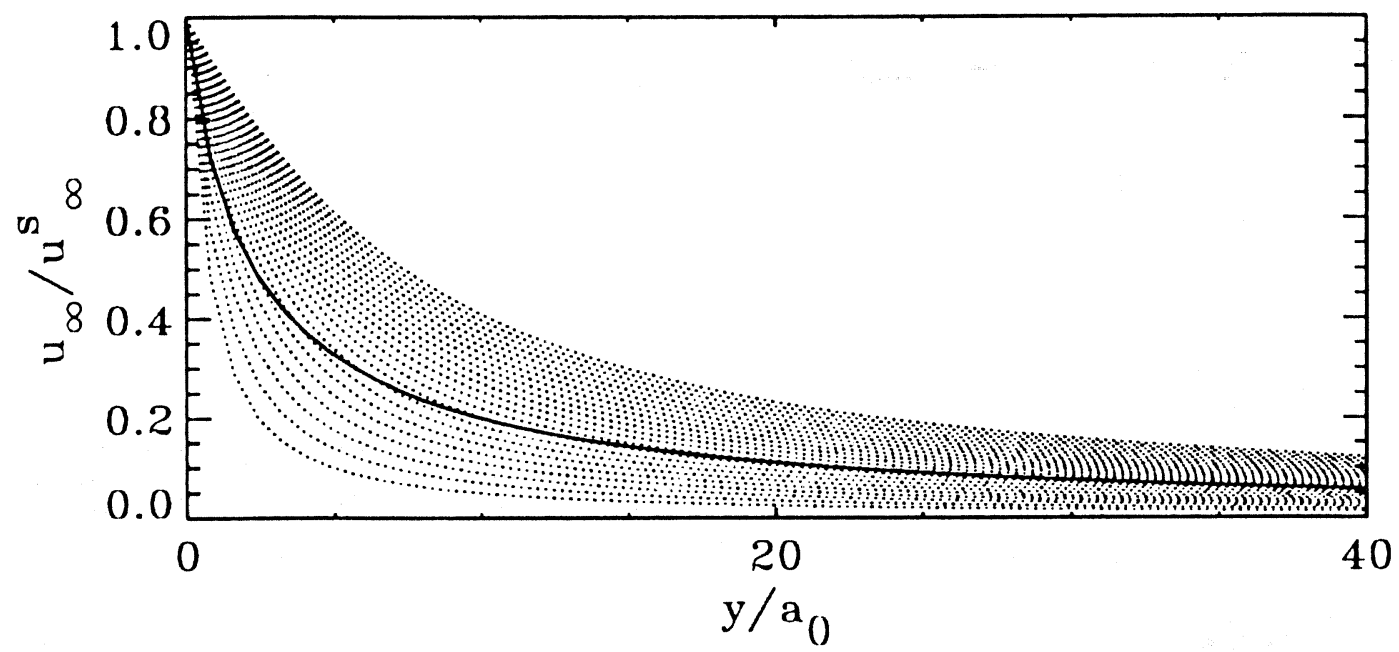

Fig. 7. Displacement parallel to the fault strike for $\tau=\infty$ along the transversal direction with respect to the fault in case of a non-singular crack (solid line) for $r_{s}=0.04$, that is a final crack extension of about $16.5 a_{0}$ and for singular cracks (dotted lines) of different extension varying between $a_{0}$ (lower curve) and $10 a_{0}$ (upper curve). Displacement is normalized to the value on the strike $u_{\infty}^{s}$ for each curve.

duced by a singular non-extending crack with rather small amplitude especially near the fault and far from the fault. We may interpret this analogy saying that the observed displacement is mainly produced by the superficial portion of the creeping region which is involved in a significant slip and is similar to that produced by a singular crack which extends down to the same depth of this portion.

\section{Discussion and conclusions}

The displacement $u_{0}$ can be associated to the fast initial relaxation of the superficial zone $x<$ $a_{0}$ assuming that in this zone the viscosity $\eta=$ $\eta_{\text {sup }}$ is null or however very little with respect to the remaining part of the fracture. In our 1991 paper in fact we have shown that it is necessary to use a high ratio between $\eta_{0}$ and the $\eta_{\text {sup }}$ (about $10^{2} \div 10^{3}$ ) in a narrow superficial zone of the fault, in order to reproduce the initial phases of the afterslip processes. This interpretation of then parameters then means that $\eta_{\text {sup }}$ and $u_{0}$ are linked to deeper features. The $T$ parameter values (and then also the $\eta_{0}$ value) are very similar in the case of Superstition Hills and Imperial Valley, which are quite near and, tectonically similar regions belonging to the same geothermal province (e.g., Lachenbruch et al., 1985). A much smaller $T$ value is instead required for the sample from Parkfield, which is a more Northern locality placed in a very different geological and geothermal context with respect to the other two cases. Finally, we may think that in both the Imperial and the Superstition Hills fault, the evolution is slower since the fracture early gets in touch with the upper astenosphere; in such zones in fact the crust is likely to be rather thin (about $23.5 \mathrm{~km}$ ) neverthless, to confirm this hipothesis, the model should be modified in order to take into account this interaction.

The model clearly is not able to reproduce the observed Parkfield and Imperial Valley data inflexions from the type curves. Such inflexions might be due to the existence of a stress asperity at depth. A yield threshold increase might be localized at that depth and to this asperity also the aftershocks on the fault might be ascribed. It is remarkable in this sense that, in the Superstition Hills case this effect is not present and the maximum aftershock concentration did not occur on 
the fault, but in the South-West zone which lies between this and the Superstition Mountain fault (Magistrale et al., 1989).

The Imperial fault and the Brawley fault region and that to which the central San Andreas fault segment, the Calaveras fault and the Hayward fault belong, represent the two regions of the San Andreas faults system where permanent creep occurs (Cohn et al., 1982). Parkfield is in a transition zone between the central section of the San Andreas fault (where mainly steady creep occurs) and the 1857 Fort Tejon earthquake section, which is presently locked. The creep rate decrease near Parkfield is ascribed by Tse et al. (1985) to the existence of a buried localized «patch», that is a slip-deficient zone, occupying a small fraction of area of the normal seismogenic zone. Also Highway 80 is in a transition zone on the Imperial fault from north-western creep to south-eastern stick-slip where the 1979 earthquake occurred (Doser and Kanamori, 1986; Cohn et al., 1982). It is then likely that the particular position of the places where Parkfield and Imperial Valley data were picked up, requires a more complex model than the present one. If one associates the inflexions to a buried asperity and imposes that the instant at which in the $r_{s}=0.01$ curve the inflexion occurs is the same of which $a(t)$ is about $10 \mathrm{~km}$, the mean aftershock depth according to, e.g., Tse et al. (1985) and Doser and Kanamori (1986), an $a_{0}$ value of the order of hundreds of meters is found. It is noticeable that Smith and Wyss (1968) estimate the depth of the alluvial layer as about half a kilometer. This layer is supposed to undergo plastic deformation and may be expected to cause the almost null coseismic effect at the Earth surface. If Scholz et al. (1969) estimate a mean depth of about $4 \mathrm{~km}$ for the seismic region top at Parkfield, he does not exclude that near Highway 46 the seismic rupture may have extended nearer to the surface. Besides on the base of 1976 observed events one may think that in the Parkfield region, an increase of the plastic threshold occurs at half a kilometer depth (Wesson, 1988). In the case of Superstition Hills, if we take an $a_{0}$ value of the order of hundreds of meters and $r_{s}=0.04$, we obtain a depth of about $(3 \div 4) \mathrm{km}$ as that in which the slip is decreased by an order of magnitude with respect to the surface. This depth is similar to that in which Scholz et al. (1969), Scholz (1991) and Wesson (1988) considered the aseismic slip to occur and this value is also suggested from the developed displacement at least at $10 \mathrm{~km}$ from the fault assuming the above value for $a_{0}$. Particularly in this model Scholz (1991) assumes that creep is confined within a superficial «velocity strengthening» layer, which can be identified with the sediments, where the dynamic friction would be greater than the static friction. At the bottom of this layer the seismic fracture stops. Assuming that in this layer the earthquake stress relaxation occurs at steady-state friction, that is, friction which depends on time only through time dependence of slip velocity, Scholz (1991) reproduces a temporal logarithmic law. Neverheless, considering the region where creep occurs as rigidly bounded is too drastic an assumption, since in this way one neglects the stress concentration due to the increasing slip, just beyond the creep region boundary. This stress concentration allows the aseismic dislocation propagation to greater depth; to oppose such an effect it is necessary to suppose that below the aseismic region a very high slip resistance exists, whereas it is likely that, in the postseismic phase, the region is weaker than it was before the earthquake, owing to slip-weakening (e.g., Dieterich, 1972) or to a dynamic friction decrease due to stick-slip (Dieterich, 1978) and that sometimes the plastic threshold and the friction may be generically interpreted as «frictional strength» of the gouge materials (Wesson, 1988). Moreover, in the fault gouge it is observed that the temperature during the earthquake may increase locally to produce partial rock melting and consequent post-seismic fault lubrication (Bossiere, 1991).

Briefly: a quasi-static non-singular crack model was proposed to describe afterslip processes on strike-slip faults. The earthquake stress relaxation on the crack is opposed by a viscous stress due to the assumed plastic rheology for the fault gouge, while the crack extension at depth is opposed by the earthquake stress drop and is favoured by crack self-stress concentration. Taking into account the presence of a very low viscosity layer near the surface (which yields a slip $u_{0}$ in the very early times) and by a model comparison with the observed later phases of afterslip, we proved that: 1) a suitable heterogeneity 
of the stress field is sufficient for data reproduction, 2) the best-fit values of the external stress parameter $r_{s}$ are less sensitive to the regional features than the values of parameters linked to rheological properties, 3) the stress parameter values are consistent with a progressive depeening of the creeping region, even if the main amount of slip is developed in the shallower fault section.

\section{Acknowledgments}

J.N. Louie and W. Thatcher are gratefully thanked for their useful suggestions and kind help in some data recovery. Work performed with financial contribution of Consiglio Nazionale delle Ricerche, GNGTS.

\section{REFERENCES}

Allen, C.R. and S.W. Smith (1966): Pre-earthquake and post-earthquake surficial displecements in the Parkfield earthquakes of June 27-29, 1966, Monterey and San Luis Obispo counties; California, Bull. Seismol. Soc. Am., 56, 966- 967.

Belardinelli, M.E. and M. Bonafede (1991): A crack model of afterslip on shallows faults, Geophys. J. Int., 106, 521-530.

BILBY, B.A. and J.D. EsHelby (1968): Dislocations and the theory of fracture, in Fracture - an Advanced Treatise, Vol. I, edited by H. Liebowitz (Academic Press, New York), pp. 99-182.

BILHAM, R. (1989): Surface slip subsequent to the 24 November 1987 Superstition Hills, California, earthquake monitored by digital creepmeters, Bull. Seismol. Soc. Am., 79, 424-450.

Bonafede, M., M. Dragoni and E. Boschi (1985): Quasistatic crack models and the frictional stress threshold criterion for slip arrest, Geophys. J. R. Astron. Soc., 83, 615-637.

Bonafede, M. and M. DRAGoni (1987): A crack model of creep processes on deep sections of faults, Geophys. J.R. Astron. Soc., 91, 1059-1086.

BossIERE, G. (1991): Petrology of pseudotachylytes from the
Alpine Fault of New Zealand, Tectonophysics, 196, 173193.

Cohn, S.N., C.R. Allen, R. Gilman and N.R. Goulty (1982): Preearthquake and postearthquake creep on the Imperial Fault and the Brawley fault zone, in the «The Imperial Valley earthquake of Oct. 15 1979», U.S. Geol. Surv. Prof. Pap., 1254, 161-167.

Crough, S.T. and R.O. Burford (1977): Empirical law for fault creep events, Tectonophysics, 42, 53-59.

DIETERICH, J.H. (1972): Time-dependent friction as a possible mechanism for aftershocks, J. Geophys. Res., 77, 33713781.

DIETERICH, J.H. (1978): Time-dependent friction and the mechanics of stick-slip, Pure Appl. Geophys., 116, 790-806.

DOSER, D.I. and H. KANAMORI (1986): Depth of seismicity in the Imperial Valley region (1977-1983) and its relationship to heat flow, crustal structure, and the October 15 , 1979, earthquake, J. Geophys. Res., 91, 675-688.

LACHENBRUCH, A.H., J.H. SASS and S.P. JR. GALANIS (1985): Heath flow in the Southernmost California and the origin of the Salton Trough, J. Geophys. Res., 90, 6709-6736.

Louie, J.N., C.R. Allen, D.C. Johnson, P.C. HAASE and S.N. CoHN (1985): Fault slip in southern California, Bull. Seismol. Soc. Am., 75, 811-833.

Magistrale, H., L. Jones and H. Kanamori (1989): The Superstition Hills, California, earthquakes of 24 November 1987, Bull. Seismol. Soc. Am., 79, 239-251.

NASON, R. and J. WEERTMAN (1973): A dislocation theory analysis of fault creep events, J. Geopys. Res., 78, 77457751 .

SCHOLZ, C.H. (1991): The mechanics of the earthquakes and faulting (Cambridge University Press, Cambridge).

ScHOLZ, C.H., M. WYSS and S.W. SMITH (1969): Seismic and aseismic slip on the San Andreas Fault, J. Geophys. Res., 74, 2049-2069.

Sharp, R.V. and J.L. SAXTON (1989): Three dimensional records of surface displacement on the Superstition Hills Fault zone associated with the earthquakes of 24 November 1987, Bull. Seismol. Soc. Am., 79, 376-389.

SMITH, S.W. and M. Wyss (1968): Displacement on the San Andreas fault subsequent to the 1966 Parkfield earthquake, Bull. Seismol. Soc. Am., 58, 1955-1973.

TSE, S.T., R. DMOWSKA and J.R. Rice (1985): Stressing of locked patches along a creeping fault, Bull. Seismol. Soc. Am., 75, 709-736.

Wesson, L.R. (1988): Dynamics of fault creep, J. Geophys. Res., 93, 8929-8951.

William, P.L. and H.W. Magistrale (1989): Slip along Superstition Hills Fault associated with the 24 November 1987 Superstition Hills, California, earthquake, Bull. Seimol. Soc. Am., 79, 390-410. 